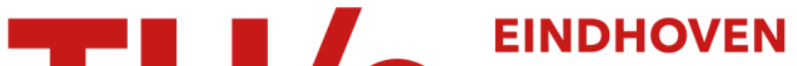 UNIVERSITY OF TECHNOLOGY
}

\section{Statistics of pressure and of pressure-velocity correlations in isotropic turbulence}

\section{Citation for published version (APA):}

Biferale, L., Gualtieri, P., \& Toschi, F. (2000). Statistics of pressure and of pressure-velocity correlations in isotropic turbulence. Physics of Fluids, 12(7), 1836-1842. https://doi.org/10.1063/1.870431

DOI:

10.1063/1.870431

Document status and date:

Published: 01/01/2000

\section{Document Version:}

Publisher's PDF, also known as Version of Record (includes final page, issue and volume numbers)

\section{Please check the document version of this publication:}

- A submitted manuscript is the version of the article upon submission and before peer-review. There can be important differences between the submitted version and the official published version of record. People interested in the research are advised to contact the author for the final version of the publication, or visit the $\mathrm{DOI}$ to the publisher's website.

- The final author version and the galley proof are versions of the publication after peer review.

- The final published version features the final layout of the paper including the volume, issue and page numbers.

Link to publication

\section{General rights}

Copyright and moral rights for the publications made accessible in the public portal are retained by the authors and/or other copyright owners and it is a condition of accessing publications that users recognise and abide by the legal requirements associated with these rights.

- Users may download and print one copy of any publication from the public portal for the purpose of private study or research.

- You may not further distribute the material or use it for any profit-making activity or commercial gain

- You may freely distribute the URL identifying the publication in the public portal.

If the publication is distributed under the terms of Article 25fa of the Dutch Copyright Act, indicated by the "Taverne" license above, please follow below link for the End User Agreement:

www.tue.nl/taverne

Take down policy

If you believe that this document breaches copyright please contact us at:

openaccess@tue.nl

providing details and we will investigate your claim. 


\title{
Statistics of pressure and of pressure-velocity correlations in isotropic turbulence
}

\author{
L. Biferale \\ Dipartimento di Fisica and INFM, Università di Tor Vergata, Via della Ricerca Scientifica 1, \\ I-00133 Roma, Italy \\ P. Gualtieri \\ Dip. di Meccanica e Aereonautica, Universitá di Roma “La Sapienza," Via Eudossiana 18, \\ 00184, Roma, Italy \\ F. Toschia) \\ University of Twente, Department of Applied Physics and J. M. Burgerscentrum for Fluid Dynamics, \\ P.O. Box 217, 7500 AE, Enschede, The Netherlands and INFM, Unitá di Tor Vergata, \\ Via della Ricerca Scientifica 1, I-00133 Roma, Italy
}

(Received 10 November 1999; accepted 15 March 2000)

Some pressure and pressure-velocity correlations in a direct numerical simulations of a three-dimensional turbulent flow at moderate Reynolds numbers have been analyzed. We have identified a set of pressure-velocity correlations which possess a good scaling behavior. Such a class of pressure-velocity correlations is determined by looking at the energy-balance across any sub-volume of the flow. According to our analysis, pressure scaling is determined by the dimensional assumption that pressure behaves as a "velocity squared," unless finite-Reynolds effects are overwhelming. The $\mathrm{SO}(3)$ decompositions of pressure structure functions has also been applied in order to investigate anisotropic effects on the pressure scaling. (C) 2000 American Institute of Physics. [S1070-6631(00)00607-3]

\section{INTRODUCTION}

Scaling in turbulent flows is one of the most challenging open issue in fluid dynamics. ${ }^{1}$ Typical problems concern both the understanding of the ideal case of isotropic and homogeneous turbulence in the limit of high-Reynolds numbers ${ }^{2,3}$ or more realistic and applied situations with anisotropic and inhomogeneous statistics (for recent examples see Refs. 4-6). In 1941, Kolmogorov, used a clever application of dimensional analysis to predict that the scaling of velocity increments in the inertial range should have a power law behavior depending only on the averaged energy dissipation in the flow, $\epsilon$. Namely, for structure function of order $p$, i.e., the $p$ th moment of a velocity difference across a distance $R$ we have

$$
S_{q}(R)=\left\langle(v(x+R)-v(x))^{q}\right\rangle \sim \epsilon^{q / 3} R^{q / 3},
$$

with $\eta \ll r \ll L_{0}$ where $\eta$ is the dissipative scale and $L_{0}$ is the typical external scale where forcing acts. Let us notice that in (1) we have explicitly neglected any tensorial structure in the velocity field such as to stress the typical dimensional character of the Kolmogorov theory, i.e., the scaling properties must be the same for any observable which has the same physical dimension and which is built in terms of local field increments, $\delta_{r} v(x)=v(x+r)-v(x)$.

Kolmogorov theory, as previously summarized, is quantitatively wrong. Experiments and numerical simulations

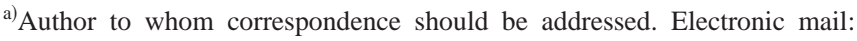
f.toschi@tn.utwente.nl
}

show a quantitative disagreement with the dimensional prediction $p / 3$ for the scaling exponents. For example, the longitudinal velocity structure functions

$$
S_{q}^{v}(r)=\left\langle\left|\left(v_{i}(\mathbf{x}+\mathbf{R})-v_{i}(\mathbf{x})\right) \hat{R}_{i}\right|^{q}\right\rangle \sim R^{\zeta_{v}(p)},
$$

show a power law behavior with a set of exponents $\zeta_{v}(p)$ nonlinear in $p$. The failure of the dimensional estimate $p / 3$ goes under the name of anomalous scaling.

Many problems naturally arise as a consequence of the failure of the main Kolmogorov prediction. The main open problem is to find an analytical way to calculate from first principle the anomalous exponents, a problem which is still out of control except for the case of anomalous exponents characterizing the statistics of passive quantities advected by Gaussian velocity fields. ${ }^{7-12}$ Another interesting question, opened by the failure of Kolmogorov dimensional prediction, consists in the possibility that local observable with the same physical dimensions but with different tensorial structures have different scaling properties. About this point, there are some experimental and numerical evidences regarding different possible anomalous behavior of longitudinal and transversal structure functions ${ }^{13-15}$ even in isotropic turbulence. Somehow related to this issue is also the apparent different anomalous scaling between the coarse grained averages of dissipative quantities like enstrophy and energy dissipation. ${ }^{16}$ On the other hand, on the basis of a $\mathrm{SO}(3)$ decomposition of velocity correlation functions, other authors ${ }^{17}$ claim that the supposed different scaling of quantities like transversal and longitudinal structure functions can only be due to spurious sub-leading nonisotropic effects, i.e., 
in isotropic high-Reynolds numbers all components of the same tensorial observable should have the same-maybe anomalous-scaling behavior. A first numerical support to this claim has been presented in the analysis of a channel flow simulation in. ${ }^{4}$

Even more complex is the situation when multipoint pressure correlations is involved. ${ }^{2,19,20}$ Dimensionally speaking pressure is just a velocity squared, and Kolmogorov-type argument can be easily generalized to the case of pressure structure functions, $F_{q}(r)$. Indeed, a simple application of dimensional analysis leads to ${ }^{21}$

$$
F_{q}(r) \equiv\left\langle|P(x)-P(x+r)|^{q}\right\rangle \sim \epsilon^{2 q / 3} r^{2 q / 3},
$$

where as usual, all distances are supposed to belong to the inertial range of scales. Of course, intermittency will also affect pressure scaling. By following the straightforward hypothesis that pressure can be treated as a velocity squared one would be tempted to assign the same intermittency exponents of the velocity field to the pressure scaling, i.e., to replace (3) with

$$
F_{q}(r) \sim r^{\zeta_{v}(2 q)}
$$

This prediction is just a simple consequence of the assumptions that all velocity correlations have the same scaling behavior supposed that all distances involved are in the inertial range and that the statistics is locally isotropic. Such a dimensional ansatz has been questioned on the basis of a phenomenological argument in Ref. 2, some numerical support to the latter argument have been recently presented in Ref. 20.

In this paper we will mainly present some numerical evidences that indeed the dimensional ansatz (4) is correct. It is well-known that this must be the case at least for $q=2$ in (4). In this case, there exist an exact relation ${ }^{22}$ which connect the scaling of the second-order pressure structure function with a linear integral combination of fourth-order velocity structure functions. The problem is if the exact result can be simply extrapolated to other pressure-dependent observable and, in the case, how strong finite-Reynolds effects can be. Indeed, one may argue that pressure feels strongly nonlocal effects, being just the inversion of the Poisson problem $\Delta P$ $=-\partial_{i} \partial_{j} v_{i} v_{j}$, and therefore, the assumptions of independence from large scales and/or from boundary conditions may not be satisfied even at very high-Reynolds numbers. Indeed, to our knowledge, neither experimental studies nor numerical simulations have ever been able to make a firm quantitative statement about pressure scaling properties. ${ }^{18-20,26}$ Pressure is also important for the understanding of many applied hydrodynamical problems like multiphase flows, flows with bubbles or sedimentation. ${ }^{23,24}$ In this paper we show that it is possible to find a set of velocity-pressure observable which have indeed a quite good scaling behavior in agreement with the dimensional ansatz (4) also at moderate Reynolds numbers.

Scaling in turbulence is particularly difficult to test in both experiments and numerical simulations. Experiments reach high-Reynolds numbers by paying the price to have a very limited set of information on the whole velocity fields, typically only a long time series of one velocity components in a few spatial points. Moreover, in most cases, there is not a precise control of the degree of isotropy and homogeneity in the flow. On the other hand, numerical simulations have a perfectly controlled lay out, the velocity field is exactly known at any point, but the maximum reachable Reynolds number is still order of magnitude smaller than in typical experiments. $^{27}$

Nevertheless, numerical simulations, if exploited in a clever way, are the only tool where complex measurements can be performed. Therefore, questions like the dependency of scaling properties from the tensorial nature of the observable can, up to now, be investigated only in a numerical data base.

In this paper, we present a detailed analysis of pressure scaling and pressure-velocity correlations scaling in a set of moderate Reynolds number simulations.

Starting from the analysis of the energy transfer in real space we propose a set of pressure-velocity observable which show better scaling properties then the usual pressure structure functions. We present quantitative evidence that indeed, while pressure structure functions are strongly affected from Reynolds numbers effects, the pressure-velocity correlation functions we investigated have a fairly good scaling behavior, even at modest Reynolds numbers, in agreement with the hypothesis that pressure "behaves" like a velocity squared. In order to understand whether the bad scaling behavior detected in the pure-pressure structure functions is due to spurious anisotropic sub-leading effects we also present some results on the $\mathrm{SO}(3)$ decomposition of the pressure field.

The paper is organized as follows. In Sec. II we summarize the known analytical result which connect the secondorder pressure structure function to the integral linear combination of fourth-order velocity correlations and the experimental and numerical attempts to test the relation. In Sec. III we introduce the set of pressure-velocity correlations which should have better scaling properties on the basis of a simple argument based on the energy transfer of NavierStokes equations in the real space. In Sec. IV we present the analysis of our numerical data base. In Sec. V we briefly comment on the analysis of nonisotropic fluctuations. Conclusions follow in Sec. VI.

\section{PRESSURE STRUCTURE FUNCTIONS}

Under the assumptions of local isotropy, local homogeneity, incompressibility, and by use of Navier-Stokes equation, one can relate the second-order pressure structure functions, $F_{2}(r)$, to some fourth-order velocity structure functions. ${ }^{22}$ Namely

$$
\begin{aligned}
F_{2}(r) \equiv & -\frac{1}{3} D_{1111}(r)+\frac{4}{3} r^{2} \int_{r}^{\infty} y^{-3}\left[D_{1111}(y)+D_{\beta \beta \beta \beta}(y)\right. \\
& \left.-6 D_{11 \gamma \gamma}\right] d y+\frac{4}{3} \int_{0}^{r} y^{-1}\left[D_{\beta \beta \beta \beta}(y)-3 D_{11 \gamma \gamma}\right] d y,
\end{aligned}
$$

where the fourth-order structure function is

$$
D_{i j k l}(\mathbf{r}) \equiv\left\langle\left(\mathbf{u}_{i}-\mathbf{u}_{i}^{\prime}\right)\left(\mathbf{u}_{j}-\mathbf{u}_{j}^{\prime}\right)\left(\mathbf{u}_{k}-\mathbf{u}_{k}^{\prime}\right)\left(\mathbf{u}_{1}-\mathbf{u}_{1}^{\prime}\right)\right\rangle,
$$


and where for simplicity we have used primed variables to express velocities at the position $\mathbf{x}^{\prime}=\mathbf{x}+\mathbf{r}$ and where $i, j, k, l$ is 1 if the velocity component is parallel to the separation vector, $\overrightarrow{\mathbf{r}}$, and 2, 3 otherwise. Subscripts $\beta, \gamma$ denote either 2 or 3. Of course, (5) implies that whenever the fourth-order structure functions entering in the above expressions are all dominated by the inertial-range intermittent scaling behavior, $D_{i, j, k, l}(r) \sim r^{\zeta_{v}(4)}$, then also the second-order pressure structure functions should scale with the exponent $\zeta_{p}(2)$ $=\zeta_{v}(4)$. Relation (5) have been carefully tested in numerical simulations without any appreciable deviations. ${ }^{18,19}$ Nevertheless, the overall scaling behavior of the pressure structure function is very poor. Similarly, the analysis of experimental data ${ }^{18}$ does not show any power law behavior for the pressure structure functions even if the Reynolds number was extremely high $\left(\operatorname{Re}_{\lambda} \sim 10000\right)$. In the latter case, authors tried to explain the difference between pressure scaling quality and velocity scaling quality by invoking a possible different scaling for the different velocity correlations entering in the rhs (right-hand side) of (5), leading to the final prediction that pressure structure functions is made in terms of different power law contributions with slightly different exponents. The resulting superposition of power laws would be the responsible of the poor observed scaling behavior. This statement would contradict the theoretical prediction made in terms of the $\mathrm{SO}(3)$ decomposition which forbids different component of the same tensorial observable to scale differently in a isotropic ensemble.

Another interesting remark consists in the strong cancellation among the different contribution of (5) observed in numerical simulations: $:^{18,19}$ The lhs (left-hand side) of (5) is more then an order of magnitude smaller than the single different contributions entering in the rhs. One, cannot exclude a priori the possibility that there exist an almost perfect cancellation of all leading scaling terms of all contribution appearing in the rhs of (5), even if such a perfect cancellation would call for some unknown physical interpretation.

More probably, the cancellation is not perfect but strong enough to hide completely the pressure scaling at the available experimental and numerical Reynolds numbers.

On the other hand, the possibility that pressureincrements behave as velocity-increments, $\delta P \sim \delta v$, instead than as a velocity-increment squared has been recently proposed. ${ }^{20}$ This would violate the exact results previously reported and, therefore, cannot be correct unless strong anisotropic effects are present at all scales.

In order to better assess the pressure statistical properties we present in the following, some results for pressuredependent observable. This observable do not possess the strong cancellation properties showed by structure function.

\section{PRESSURE-VELOCITY CORRELATION}

Let us start by looking at the energy balance inside any volume $V$ of the flow. From the Navier-Stokes (NS) equations we obviously have

$$
\begin{gathered}
\partial_{t} E_{V}+\int_{V} v_{i}(\mathbf{x}) v_{j}(\mathbf{x}) \partial_{j} v_{i}(\mathbf{x}) d \mathbf{x}+\int_{V} v_{i}(\mathbf{x}) \partial_{i} P(\mathbf{x}) d \mathbf{x} \\
=\nu \int_{V} v_{i}(\mathbf{x}) \Delta v_{i}(\mathbf{x}) d \mathbf{x},
\end{gathered}
$$

where with $E_{V}=\frac{1}{2} \int_{V} d \mathbf{x} v_{i} v_{i}$ we denote the total energy in the sub-volume $V$. Let us notice that the two terms in the lhs of (6) can be obviously written as the fluxes across the boundaries of $V$ by using Gauss theorem

$$
\begin{aligned}
\int_{V} v_{i}(\mathbf{x}) v_{j}(\mathbf{x}) \partial_{j} v_{i}(\mathbf{x}) d \mathbf{x} \equiv \frac{1}{2} \int_{\Omega V} d \Sigma n_{j} v_{j}(\mathbf{x}) v^{2}(\mathbf{x}) \\
\equiv \Phi_{\Omega_{V}}\left(\mathbf{v} v^{2}\right), \\
\int_{V} v_{i}(\mathbf{x}) \partial_{i} P(\mathbf{x}) d \mathbf{x} \equiv \int_{\Omega_{V}} d \Sigma n_{i} v_{i}(\mathbf{x}) P(\mathbf{x}) \equiv \Phi_{\Omega_{V}}(\mathbf{v} P),
\end{aligned}
$$

where with $n_{i}$ we denote the unit vector perpendicular to the infinitesimal surface on the boundaries of $V$. Rewritten in this way, relation (6) is just a simple restatement of the conservation of energy: The total energy change inside a volume is given by the flux across the volume surface and by the energy dissipation inside the volume. Let us now use this simple fact in order to extract some useful considerations about scaling properties of velocity-pressure correlations. Let us consider a very particular class of volume $V$, i.e., a cylinder with an infinitesimal squared basis of surface $\delta$ and with a finite axis in the direction of $\mathbf{R}$. In the limit when the basis becomes smaller and smaller, the flux across the lateral sides goes to zero because contributions from two opposite walls are equal but with different signs. The only contributions to the total flux come from the two infinitesimal basis and can be written as

$$
\Phi_{\Omega_{V}}\left(\mathbf{v} v^{2}\right)=\delta\left(v_{i}(\mathbf{x}) v^{2}(\mathbf{x})-v_{i}(x+\mathbf{R}) v^{2}(\mathbf{x}+\mathbf{R})\right) \hat{R}_{i},
$$

for the flux involving the velocity correlation and as

$$
\Phi_{\Omega_{V}}(\mathbf{v} P)=\delta\left(v_{i}(\mathbf{x}) P(\mathbf{x})-v_{i}(\mathbf{x}+\mathbf{R}) P(\mathbf{x}+\mathbf{R})\right) R_{i},
$$

for the flux involving pressure-velocity correlations. In both cases we have exploited the fact that the two infinitesimal basis are centered in $\mathbf{x}$ and in $\mathbf{x}+\mathbf{R}$, i.e., their unit vector is oriented along $\hat{R}$. Similarly the two volume integral giving the time variation of the total energy and the energy dissipation becomes two linear integral times the infinitesimal basis area $\delta: \partial_{t}\left(\delta \int_{\mathbf{R}} d s v^{2}(\mathbf{x})\right)$ and $\left(\delta \int_{\mathbf{R}} d s v_{i}(\mathbf{x}) \Delta v_{i}(x)\right)$ respectively, where with $d s$ we parameterized the segment going from $\mathbf{x}$ to $\mathbf{x}+\mathbf{R}$.

Let us now assume that all the four observable entering in the energy balance have the same statistical behavior. This is somehow a "local Kolmogorov refined hypothesis:" We link the scaling of the local energy dissipation to the scaling of some particular third-order velocity correlation and to the scaling of a velocity-pressure correlation. The claim is, therefore, that the particular structure functions emerging from our flux analysis should scale exactly like the coarse grained energy dissipation, i.e., should have an anomalous scaling like the usual longitudinal structure functions which satisfy 


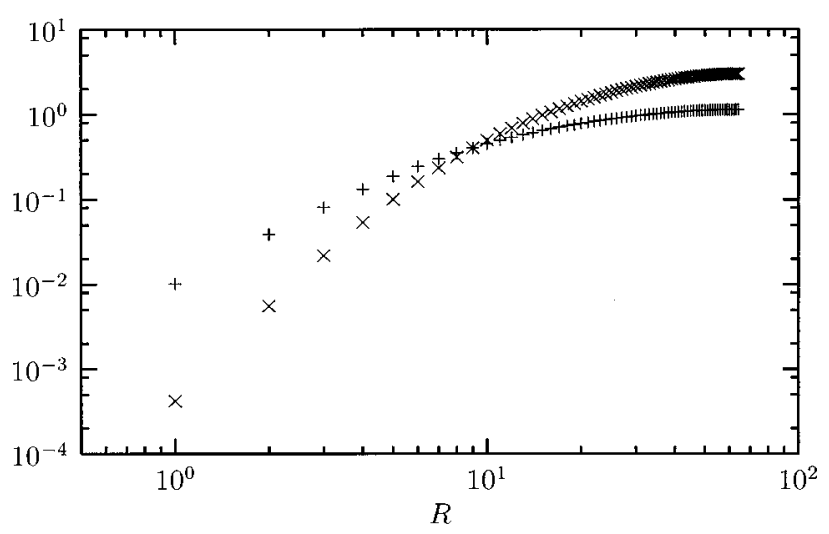

FIG. 1. $\log -\log$ plot of $F_{1}(R)(+)$ and of $S_{2}(R),(\times)$. Notice that the pressure structure function is about three times smaller than the velocity structure function at large scale.

the original Kolmogorov Refined Hypothesis. In the next section we present some numerical data in support of this claim.

\section{NUMERICAL ANALYSIS}

The data set we are going to analyze has been obtained from a direct numerical integration of NS equations using a pseudo-spectral method with dealiazing on a grid of $128^{3}$ points. The forcing was implemented isotropically on all wave vectors with $|k|<1$ such as to enforce the $k^{-5 / 3}$ spectrum at small wave vectors. ${ }^{25}$ We have analyzed about 100 configurations stored each eddy-turn over time. The simulation has a Reynolds ${ }_{\lambda}=70$. The ratio between the highest resolved wave number $k_{\max }$ and the Kolmogorov scale $k_{\eta}$ is $K_{\max } / k_{\eta}=1.5$, which is within the usual accepted range to ensure a stable code and a fairly good resolution.

Let us denote with:

$$
\begin{aligned}
& S_{q}^{v v^{2}}(R)=\left\langle\left|\left(v_{i}(\mathbf{x}) v^{2}(\mathbf{x})-v_{i}(\mathbf{x}+\mathbf{R}) v^{2}(\mathbf{x}+\mathbf{R})\right) \hat{R}_{i}\right|^{q / 3}\right\rangle, \\
& S_{q}^{v P}(R)=\left\langle\left|\left(v_{i}(\mathbf{x}) P(\mathbf{x})-v_{i}(\mathbf{x}+\mathbf{R}) P(\mathbf{x}+\mathbf{R})\right) \hat{R}_{i}\right|^{q / 3}\right\rangle,
\end{aligned}
$$

the two different structure functions which can be made in terms of the two flux quantities defined in the previous section. Let us notice that in (11) both $S_{q}^{v v^{2}}(R)$ and $S_{q}^{v P}(R)$ have been defined as the $q / 3$ power of the original fluxes such as to have the same dimensions of $S_{q}^{v}(R)$.

Let us start by showing in Fig. 1 the strong cancellation effects present in the pressure structure functions $F_{q}(R)$ with respect to the velocity longitudinal structure functions with the same physical dimensions $S_{2 q}^{v}(R)$. In Fig. 1 we show the $\log -\log$ plot of $F_{1}(R)$ and of $S_{2}(R)$, as one can see the overall amplitude of pressure fluctuations is about three times smaller than the velocity fluctuations. This is just to confirm that pressure by itself is a much weaker signal than the usual velocity correlations.

As one can see in Fig. 1 the scaling is quite poor, as one can expect in any DNS (direct numerical simulation). As usual, in order to extract quantitative statement about scaling exponents one has to exploit the extended self similarity (ESS) property enjoyed by homogeneous and isotropic tur-

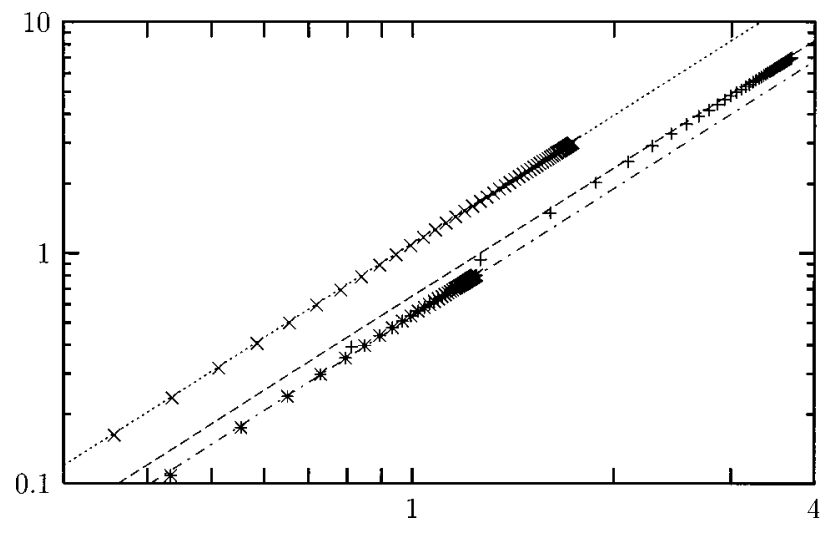

FIG. 2. ESS $\log -\log$ plot of $S_{2}^{v v^{2}}(R)$ vs $S_{1}^{v v^{2}}(R),(+) ; S_{2}^{v P}(R)$ vs $S_{1}^{v P}(R)$, $(\star) ; S_{2}^{v}(R)$ vs $S_{1}^{v}(R),(\times)$; superimposed are the straight lines with the isotropic and homogeneous high-Reynolds slope, $\zeta_{2}^{v} / \zeta_{1}^{v}=1.92$.

bulent flows. ${ }^{28}$ ESS consists in looking for relative scaling of two different observable. Usually, one takes two structure functions of two different orders, i.e., in the case of longitudinal structure functions $S_{q}^{v}(R) \sim\left[S_{q^{\prime}}^{v}(R)\right]^{\zeta_{v}(q) / \zeta_{v}\left(q^{\prime}\right)}$.

Let us now define the same relative scaling for the two generalized structure functions defined in (11).

$$
\begin{aligned}
& S_{q}^{v v^{2}}(R) \sim\left[S_{q^{\prime}}^{v v^{2}}(R)\right]^{\zeta_{v v^{2}}(q) / \zeta_{v v^{2}}\left(q^{\prime}\right),} \\
& S_{q}^{v P}(R) \sim\left[S_{q^{\prime}}^{v P}(R)\right]^{\zeta_{v P}(q) / \zeta_{v P}\left(q^{\prime}\right) .}
\end{aligned}
$$

In Fig. 2, we show the ESS plot for the two generalized structure functions and for the usual longitudinal structure functions, respectively, with $q=1, q^{\prime}=2$. As one can see all the ESS plots showed a scaling behavior consistent with the usual homogeneous and isotropic high Reynolds value which give for the relative exponents: $\zeta_{v}(2) / \zeta_{v}(1)=1.92 \pm 0.02 .{ }^{28}$ Similar agreements are found for higher-order moments (not showed).

The scaling ansatz assumed for the generalized structure functions seems, therefore, quite well satisfied. These findings support the fact that pressure does not behave abnormally as far as its "dimensional" scaling properties are concerned. Indeed, pressure-velocity correlations behaves exactly like velocity-velocity correlations once pressure is counted as a "velocity squared." Nevertheless, the only realistic way to perform a quantitative statement about scaling exponents is to study the logarithmic local slopes of (12). Only when logarithmic local slopes show a fairly constant behavior one can really speak about scaling. In Fig. 3 we show the logarithmic local slopes of (12) for $q=1, q^{\prime}=2$ together with the corresponding quantities measure for the longitudinal velocity structure functions. In order to show that the hypothesis that pressure-increments behave as a linear velocity-increment, $\delta P \sim \delta v$, is definitely ruled out by our data we also show in Fig. 3 the logarithmic local slope of the ESS applied to pressure structure-functions for $q=1$, $q^{\prime}=2$. As one can see, while the three slopes measured on the flux structure functions and on the longitudinal structure functions agree perfectly with the high-Reynolds numbers measurements, the pure-pressure structure functions is defi- 


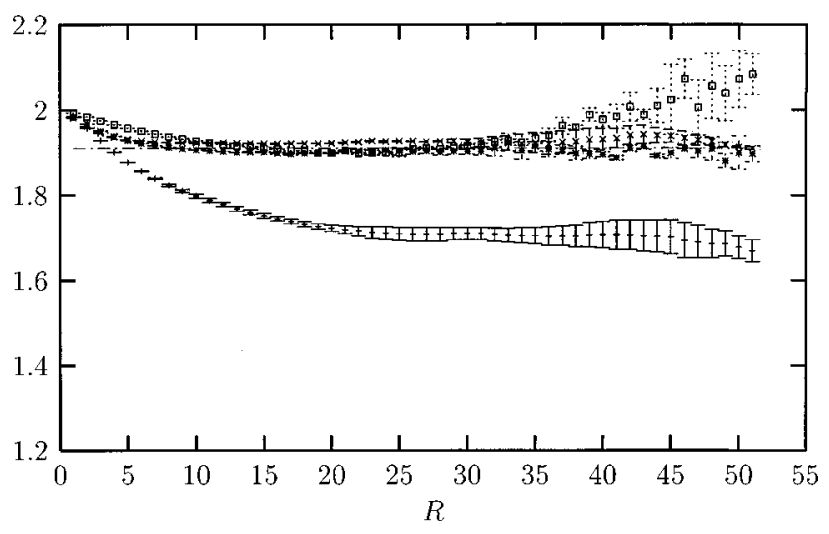

FIG. 3. Logarithmic local slope of: $(*) S_{q}^{v v^{2}}(R)$ vs $S_{q^{\prime}}^{v v^{2}}(R)$; $(\square) S_{q}^{v P}(R)$ vs $S_{q^{\prime}}^{v P}(R) ;(\times) S_{q}^{v}(R)$, vs $S_{q^{\prime}}^{v}(R)$, for $q=2$ and $q^{\prime}=1$. Notice that all the above values are in perfect agreement with the high-Reynolds number value 1.92 (straight line), while the logarithmic local slope for the pure-pressure structure functions $F_{2}(R)$ vs $F_{1}(R),(+)$ is different. The error bars are estimated by looking at the fluctuations over the first half and the second half of the whole set of configurations.

nitely much poorer. In Fig. 4 we plot the same as Fig. 3 but for a different choice of moments, $q=2, q^{\prime}=4$, for both fluxes and longitudinal structure functions and with $q=1$, $q^{\prime}=2$ for the pure pressure structure functions. In this way we are comparing quantities with exactly the same dimensional properties. Again, while the flux-made structure functions, $S_{q}^{v P}(R), S_{q}^{v v^{2}}(R)$ and the longitudinal structure functions, $S_{q}^{v}(R)$ have the same local slope the pure-pressure result obtained on the ESS of $F_{q}(R)$ shows a poorer and different scaling.

A few comments are now in order. On one hand, we see from Figs. 3 and 4 that the simple local-refined Kolmogorov hypothesis derived in the previous section is correct, i.e., fluxes (11) have the same scaling properties of the usual longitudinal structure function in homogeneous and isotropic turbulence, confirming that these observable with the same

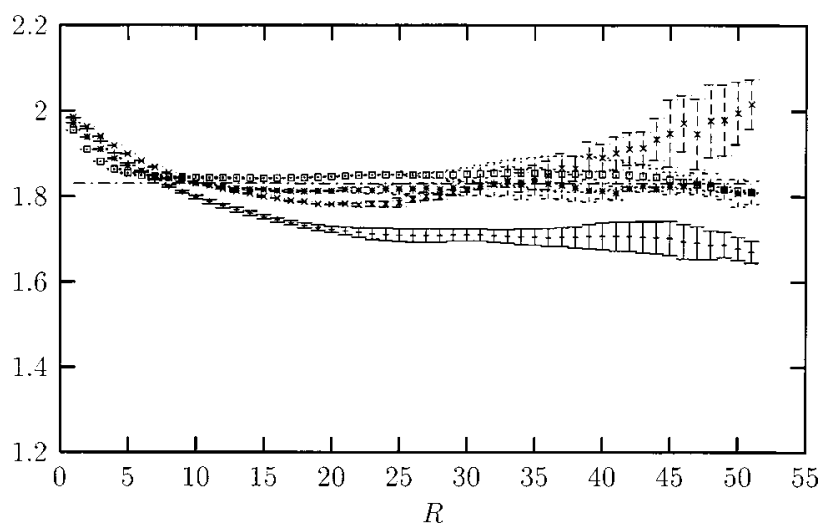

FIG. 4. Logarithmic local slopes of: $(\square) S_{q}^{v v^{2}}(R)$ vs $S_{q^{\prime}}^{v v^{2}}(R)$; (*) $S_{q}^{v P}(R)$ vs $S_{q^{\prime}}^{v P}(R) ;(\times) S_{q}^{v}(R)$, vs $S_{q^{\prime}}^{v}(R)$, for $q=4$ and $q^{\prime}=2$. Notice that, as in Fig. 2 , the flux-based structure functions have the same scaling behavior of the longitudinal structure function in agreement with the high-Reynolds regime (straight line). Here we present a comparison with the Pressure structure function with the same physical dimensions of the flux-based structure functions, i.e., $F_{2}(R)$ vs $F_{1}(r),(+)$, still the pure-pressure structure function seems to have a different local slope. physical dimensions and built in terms of local field increments scale in the same way. On the other hand, (see Fig. 4) pure-pressure structure functions seem to violate the previous statement despite of the fact that in this case there even exist an exact result (5) supporting it. Why pure-pressure correlations show this strong deviation from the straightforward dimensional estimate? One possible explanation is connected to the-possible-lack of isotropy in the statistics. Any isotropically forced DNS is affected by possible nonisotropic fluctuations induced by the discretization of the numerical grid. In the following section we have analyzed nonisotropic effects on both velocity and pressure fluctuations.

\section{ANISOTROPIC EFFECTS}

The exact relation which connect the second-order pressure structure function with a linear integral combination of fourth-order velocity structure function (5) is correct only in the isotropic and homogeneous case. In order to test the degree of isotropy of our simulation we have proceeded in a systematic decomposition in terms of the irreducible representations of the $\mathrm{SO}(3)$ symmetry group., ${ }^{4,6,17} \mathrm{The} \mathrm{SO}(3)$ decomposition is particularly simple to apply to scalar observable, i.e., observable with all vectorial indexes contracted, like pressure structure functions or longitudinal structure functions on the kind analyzed in this work. In these case, the $\mathrm{SO}(3)$ decomposition is nothing but a decomposition in spherical harmonics. For example, the longitudinal structure functions, $\left.S_{q}^{v}(\mathbf{R})=\left\langle\left(v_{i}(\mathbf{x}+\mathbf{R})-v_{i}(\mathbf{x})\right) \hat{R}_{i}\right)^{q}\right\rangle$, can be decomposed as

$$
S_{q}^{v}(\mathbf{R}) \equiv \sum_{j m} S_{q}^{j m}(R) Y_{j m}(\hat{\mathbf{R}}),
$$

where now, we have explicitly considered the possibility that the undecomposed structure functions depend on the whole vector $\mathbf{R}$ and not only on its magnitude as in the previous sections when isotropy was assumed. The coefficient of the decomposition, $S_{q}^{j m}(R)$ depend only on the magnitude of $R$ and on the two "quantum" numbers $j, m$ which labels the properties under rotations of the $Y_{j m}$ eigenfunction. Obviously, in the case of perfect isotropy we would have only one projection alive, i.e., the projection on $Y_{00}$. The $\mathrm{SO}(3)$ decomposition here summarized has been already used in some experimental and numerical data analysis to properly disentangle the anisotropic effects from the isotropic ones. ${ }^{4,6,12,17}$ In our case, the relative amplitudes of $S_{q}^{00}(|R|)$ with respect to the anisotropic fluctuations $S_{q}^{j m}(|R|)$ with $j>0,-j \leqslant m$ $\leqslant j$, gives a direct quantitative estimate of the degree of anisotropic fluctuations for any scale $|R|$. In Fig. 5 we show the $\log -\log$ plot of the undecomposed second-order longitudinal structure functions and of its projection on the fully isotropic eigenfunction $Y_{00}$. As it is possible to see, despite of the isotropic forcing used in the simulation, the finite-size effects introduced by our computational grid are quite important at large scales: The projection, $S_{2}^{00}(|R|)$, shows a definitely better scaling than the undecomposed structure function already as a function of the real separation $R$, i.e., without using ESS. This dramatic effect was already ob- 


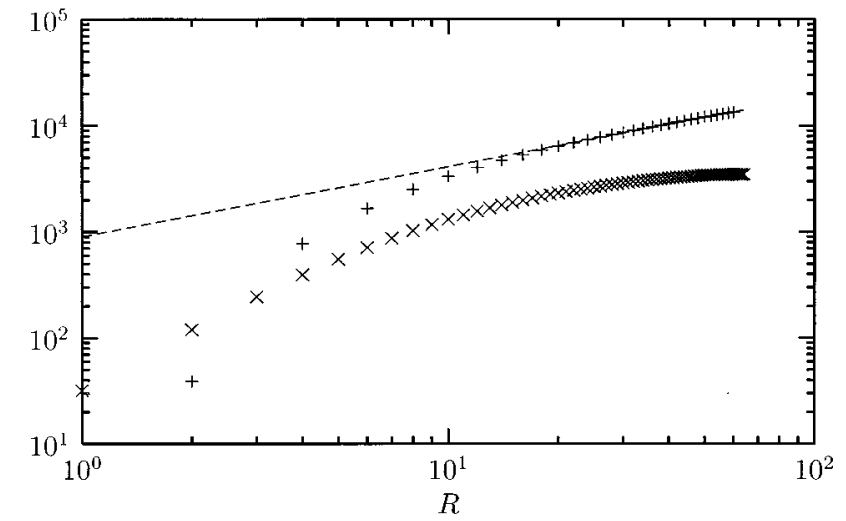

FIG. 5. Undecomposed second-order velocity structure functions $S_{2}^{v}(\mathbf{R})$ measured on the plane $x-y,(\times)$; and the projection, $S_{2}^{00}(R)(+)$, on the isotropic eigenfunction. The straight line has the high-Reynolds slope $\zeta_{2}^{v}$ $=0.7$. Notice that already in the $R$-space, the $\mathrm{SO}(3)$ decomposition improve the overall scaling behavior. The two curves have been shifted along the $y$ axis for the sake of presentation.

served in a similar application to the decomposition of velocity fluctuations inside a channel. ${ }^{4}$ Figure 5 definitely show that the $\mathrm{SO}(3)$ decomposition can help in cleaning scaling properties also in "quasi-isotropic" simulations.

On the other hand, the situation is quite different when the same decomposition is applied to the pressure structure functions

$$
F_{q}^{v}(\mathbf{R}) \equiv \sum_{j m} F_{q}^{j m}(R) Y_{j m}(\hat{\mathbf{R}}) .
$$

Let us notice that pressure is a quasi-isotropic observable also for strong anisotropic velocity configuration. Indeed, being the solutions of a Poisson problem, pressure is always an average of velocity fluctuations on all spatial directions. This simple considerations is perfectly verified on our numerical simulation. In Fig. 6 we show the undecomposed secondorder pressure structure function together with its projection on the fully isotropic harmonics, $F_{q}^{00}(R)$. One can hardly detect any differences, suggesting that anisotropic fluctua-

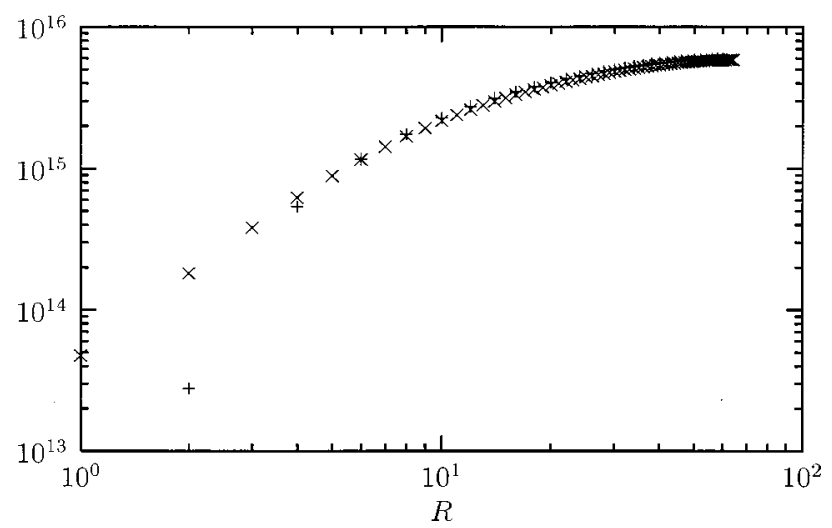

FIG. 6. Undecomposed second-order pressure structure functions $F_{2}(\mathbf{R})$ measured on the plane $x-y,(\times)$; and the projection, $F_{2}^{00}(R)(+)$, on the isotropic eigenfunction. Notice that, at difference from Fig. 5, here the decomposed and undecomposed structure functions are almost identical, indicating a high degree of isotropization in the pressure statistics. tions cannot be responsible for the poor scaling observed in the previous sections for the pure-pressure structure functions.

The only possibility to reconcile the exact result (5) with the poor scaling agreement between pressure structure functions and velocity structure functions is, in our opinion, to invoke strong Reynolds effects, i.e., in order to see a good scaling for pressure one needs to go to very high Reynolds. Indeed, the resolution of the Poisson problem certainly introduces strong nonlocal effects on the statistics. Nonlocality may also translate in strong long-range effects in the Fourier space as far as the importance of boundary conditions and forcing on the inertial range properties are concerned. If this is correct, there is no reason to expect good scaling properties for pure-pressure correlations, unless the Reynolds number is high enough to recover also in laboratory experiments an almost "infinite" inertial range extension.

\section{CONCLUSIONS}

We have analyzed some pressure and pressure-velocity correlations in a Direct Numerical Simulations at moderate Reynolds numbers. We have derived on the basis of a simple analysis of energy transfer properties across any sub-volume in the real space what we call a "local" -Refined Kolmogorov Hypothesis. We have identified a set of pressurevelocity correlations which should have a good scaling behavior because connected via the local-RKH to the scaling of the energy dissipation coarse grained on inertial range scales.

We have showed that our scaling hypothesis is well verified, while pure pressure correlations feel strong Reynolds effects. According to our analysis pressure scaling is perfectly determined by the dimensional assumption that pressure behaves as a "velocity squared," unless the finiteReynolds effects are overwhelming. We do not find any sign which could support the fact that pressure differences behave as velocity differences as proposed in Ref. 20.

We have also applied the $\mathrm{SO}(3)$ decompositions to the pressure structure functions in order to show that poor scaling properties showed by pure-pressure structure functions are not connected to anisotropic fluctuations.

\section{ACKNOWLEDGMENTS}

We acknowledge useful remarks from D. Lohse and O. Boratav. We acknowledge I. Mazzitelli for helping in the $\mathrm{SO}$ (3) analysis. L. B. and F. T. have been partially supported by INFM (PRA-TURBO) and by the EU contract FMRX CT98-0175.

${ }^{1}$ U. Frisch, Turbulence: the Legacy of A. N. Kolmogorov (Cambridge University Press, Cambridge, UK, 1995).

${ }^{2}$ M. Nelkin, "Universality and scaling in fully developed turbulence," Adv. Phys. 43, 143 (1994).

${ }^{3}$ K. R. Sreenivasan and R. A. Antonia, "The phenomenology of small-scale turbulence," Annu. Rev. Fluid Mech. 29, 435 (1997).

${ }^{4}$ I. Arad, L. Biferale, I. Mazzitelli, and I. Procaccia, "Disentangling scaling properties in anisotropic and inhomogeneous turbulence,' Phys. Rev. Lett. 82, 5040 (1999).

${ }^{5}$ F. Toschi, G. Amati, S. Succi, R. Piva, and R. Benzi, "Intermittency and structure functions in channel flow turbulence," Phys. Rev. Lett. 82, 5044 (1999). 
${ }^{6}$ I. Arad, B. Dhruva, S. Kurien, V. S. L'vov, I. Procaccia, and K. R. Sreenivasan, "Extraction of anisotropic contributions in turbulent flows," Phys. Rev. Lett. 81, 5330 (1998).

${ }^{7}$ R. H. Kraichnan, "Anomalous scaling of a randomly advected passive scalar,” Phys. Rev. Lett. 72, 1016 (1994).

${ }^{8} \mathrm{~K}$. Gawedski and A. Kupiainen, “Anomalous scaling of the passive scalar,' Phys. Rev. Lett. 75, 3834 (1995).

${ }^{9}$ M. Vergassola, “Anomalous scaling for passively advected magnetic fields,’ Phys. Rev. E 53, R3021 (1996).

${ }^{10}$ A. Lanotte and A. Mazzino, "Anisotropic nonperturbative zero modes for passively advected magnetic fields," Phys. Rev. E 60, R3483 (1999).

${ }^{11}$ R. Benzi, L. Biferale, and A. Wirth, "Analytic calculation of anomalous scaling in random shell models of passive scalars," Phys. Rev. Lett. 78, 4926 (1997).

${ }^{12}$ I. Arad, L. Biferale, and I. Procaccia, "Nonperturbative spectrum of anomalous scaling exponents in the anisotropic sectors of passively advected magnetic fields," Phys. Rev. E 61, 2654 (2000).

${ }^{13}$ S. Grossmann, D. Lohse, and A. Reeh, "Different intermittency for longitudinal and transversal turbulent fluctuations," Phys. Fluids 9, 3817 (1997).

${ }^{14}$ O. N. Boratav, "On longitudinal and lateral moment hierarchy in turbulence,"’ Phys. Fluids 9, 3120 (1997).

${ }^{15}$ B. Dhruva, Y. Tsuji, and K. R. Sreenivasan, "Transverse structure functions in high-Reynolds-number turbulence," Phys. Rev. E 56, R4928 (1997).

${ }^{16}$ S. Chen, K. R. Sreenivasan, M. Nelkin, and N. Cao, "Refined similarity hypothesis for transverse structure functions in fluid turbulence," Phys. Rev. Lett. 79, 2253 (1997).
${ }^{17}$ I. Arad, V. S. L'vov, and I. Procaccia, " Correlation functions in isotropic and anisotropic turbulence: the role of the symmetry group," Phys. Rev. E 59, 6753 (1999)

${ }^{18}$ M. Nelkin and S. Chen, "The scaling of pressure in isotropic turbulence," Phys. Fluids 10, 2119 (1998).

${ }^{19}$ R. G. Hill and O. N. Boratav, "Pressure statistics for locally isotropic turbulence," Phys. Rev. E 56, R2363 (1997).

${ }^{20}$ N. Cao, S. Chen, and G. D. Doolen, "Statistics and structures of pressure in isotropic turbulence," Phys. Fluids 11, 2235 (1999).

${ }^{21}$ A. S. Monim and A. M. Yaglom, Statistical Fluid Mechanics, Vol. 2 (M.I.T Press, Cambridge, 1975).

${ }^{22}$ R. J. Hill and J. M. Wilczak, "Pressure structure functions and spectra for locally isotropic turbulence," J. Fluid Mech. 296, 247 (1995).

${ }^{23} \mathrm{P}$. Vedula and P. K. Yeung, "Similarity scaling of accelaration and pressure statistics in numerical simulations of isotropic turbulence,' Phys. Fluids 11, 1208 (1999).

${ }^{24}$ S. Douday, Y. Couder, and M. E. Brachet, "Direct observation of the intermittency of intense vorticity filaments in turbulence,' Phys. Rev. Lett. 67, 983 (1991).

${ }^{25}$ M. R. Overholt and B. Pope, "A deterministic forcing scheme for direct numerical simulation of turbulence," Comput. Fluids 27, 1 (1998).

${ }^{26}$ M. Lesieur, Turbulence in Fluids, 3rd ed. (Kluwer Academic, 1997).

${ }^{27} \mathrm{~S}$. Y. Chen and X. Shan, "High resolution turbulent simulations using the connectin machine-2," Comput. Phys. 6, 643 (1992).

${ }^{28}$ R. Benzi, S. Ciliberto, R. Tripiccione, C. Baudet, F. Massaioli, and S. Succi, "Extended self similarity in turbulent flows," Phys. Rev. E 48, R29 (1993). 